\title{
Revisión del constructo y fundamentos teóricos de la alimentación emocional
}

\author{
Review of the construct and theoretical fundations of emotional eating
}

\author{
Vanessa M. Vázquez-Vázquez ${ }^{a}$, Lilián E. Bosques-Brugada ${ }^{b}$, Rebeca M. E. Guzmán-Saldaña ${ }^{c}$, \\ Angélica Romero-Palencia ${ }^{d}$, Karina Reyes-Jarquín ${ }^{e}$, Karina Franco-Paredes $^{f}$
}

\begin{abstract}
:
Emotional eating (EE) generally is described as a type of food intake emitted in response to emotions, mainly unpleasant, currently it has received more attention because for associations that have been encountered with health problems, such as overweight and obesity, metabolic syndrome as well as feeding and eating disorders (mainly binge eating). However, EE concept has been analyzed and questioned little about its own conceptualization, theoretical background and measurement. In this document emotional eating's main conceptualizations, from psychometric instruments and theories, are exposed. In this review, some questions that arise refer to: (1) if EE necessarily implies an overeating and/or loss of control, indicative of a probable presence of binge eating episodes; (2) how much the choice of types food (for example: sweet, salty, bitter, spicy, fatty or high in fiber) is independent, as long as it's use as a coping strategy; (3) what extent the conceptual definition should include all emotions, regardless of their nature (positive or negative) and; (4) which of the terms in spanish: "alimentación emocional", "comer por compensación psicológica" and "sobreingesta por compensación psicológica", is more attached to the construct original of emotional eating. Based on the foregoing, EE can be seen as a more complex term than has been defined so far, and that requires greater reflection, study and integration of new knowledge in its theoretical meaning and conceptual delimitation, which allows more accurate interpretations of the implications of this type of diet with a view to future researches and interventions in favor of physical and psychological health that are related to EE.
\end{abstract}

Keywords:

Emotional eating, health problems, conceptualization, psychometric instruments, theories

Resumen:

La alimentación emocional (AE) generalmente descrita como un tipo de ingesta alimentaria emitida en respuesta a emociones, principalmente desagradables, actualmente ha recibido mayor atención a razón de las asociaciones que se han encontrado con afecciones a la salud, como es el caso del sobrepeso y la obesidad, el síndrome metabólico al igual que los trastornos alimentarios y de la ingesta de alimentos (principalmente con el trastorno por atracón). Sin embargo, poco se ha analizado y cuestionado sobre su propia conceptualización, antecedentes teóricos y medición, por lo que en el presente documento se exponen las principales conceptualizaciones de la $\mathrm{AE}$ de instrumentos psicométricos y teorías. En esta revisión algunos cuestionamientos que surgen refieren a: (1) si necesariamente la AE implica una sobreingesta de alimentos y/o la pérdida de control, indicativo de una probable presencia de episodios de atracón; (2) qué tanto la elección de los tipos de alimentos (por ejemplo: dulces, salados, amargos, picantes, grasos o con alto contenido en fibra) es independiente, mientras su uso sea como una estrategia de afrontamiento; (3) en qué medida la definición conceptual debe incluir todas las emociones, independientemente de su naturaleza (positivas o negativas)

\footnotetext{
a Autor de Correspondencia, Universidad Autónoma del Estado de Hidalgo, Instituto de Ciencias de la Salud (ICSA), San Agustín Tlaxiaca, Hgo., México. https://orcid.org/0000-0003-2357-4157, Email: psic.hgo.vanessa94@ gmail.com

b Universidad Autónoma del Estado de Hidalgo, Instituto de Ciencias de la Salud (ICSA), San Agustín Tlaxiaca, Hgo., México. https://orcid.org/0000-0002-3969-683X,'Email: lilian_bosques@uaeh.edu.mx

Universidad Autónoma del Estado de Hidalgo, Instituto de Ciencias de la Salud (ICSA), San Agustín Tlaxiaca, Hgo., México. https://orcid.org/0000-0003-0877-4871, Email: remar64@yahoo.com.mx

d Universidad Autónoma del Estado de Hidalgo, Instituto de Ciencias de la Salud (ICSA), San Agustín Tlaxiaca, Hgo., México. https://orcid.org/0000-0003-0933-6937, Email: aacrom@gmail.com

${ }^{e}$ Universidad Autónoma del Estado de Hidalgo, Instituto de Ciencias de la Salud (ICSA), San Agustín Tlaxiaca, Hgo., México. https://orcid.org/0000-0003-3660-3594, Email: krjarquin@ gmail.com

${ }^{\mathrm{f}}$ Universidad de Guadalajara, Centro Universitario del Sur (CUSur), Jalisco, GDL., México. https://orcid.org/0000-0002-5899-3071, Email: karina.franco@academicos.udg.mx
} 
y; (4) cuál de los términos en español: alimentación emocional, comer por compensación psicológica y sobreingesta por compensación psicológica, se apega más al constructo original de "emotional eating". A razón de lo anteriormente expuesto se observa que, la $\mathrm{AE}$ es un término más complejo de lo que hasta ahora se ha definido, y que requiere una mayor reflexión, estudio e integración de los nuevos conocimientos en su acepción teórica y delimitación conceptual, que permita interpretaciones más acertadas de las implicaciones de este tipo de alimentación con miras de futuras investigaciones e intervenciones en favor de la salud física y psicológica que estén relacionadas con la AE.

Palabras Clave:

Alimentación emocional, problemas de salud, conceptualización, instrumentos psicométricos, teorías

\section{Introducción}

El comportamiento alimentario es visto como un constructo multidimensional, más allá de la satisfacción de necesidades meramente fisiológicas [1]. En México, se ha encontrado que la población adulta asocia la alimentación principalmente con las emociones (predominando las referentes a estados afectivos negativos), el ambiente y la cultura; reflejada en la convivencia, los eventos especiales, la familia, etcétera [2].

Específicamente, se han reportado que las emociones son uno de los factores que tienen la capacidad de modificar los patrones alimentarios (tanto en animales como humanos), a partir de la naturaleza propia del estado afectivo del que se trate, así como de las características personales [3].

El concepto de alimentación emocional (AE) emergió de teorías principalmente psicodinámicas, que buscaban dar una explicación psicológica de la obesidad, abordando el rol de las emociones para incrementar el consumo de alimentos [4], se tiene detectado que este constructo se midió por primera vez en el Cuestionario Holandés de Conducta Alimentaria (DEBQ, por sus siglas en inglés [5]), en el cual se hace alusión a la ingesta de alimentos en respuesta a emociones generalmente desagradables, y en ausencia de hambre fisiológica. Los autores de este instrumento se fundamentaron en la teoría psicosomática, que aborda el empleo de los alimentos como una forma de lidiar con la angustia percibida, ello principalmente en personas con exceso de peso. Sin embargo, antes de este instrumento, el Cuestionario de Tres Factores de la Alimentación (TFEQ, por sus siglas en inglés [6]) en su versión original evaluaba entre sus factores la desinhibición alimentaria, que posteriormente, en la revisión realizada por Ganley [3] se subdividió en dos subescalas, siendo nombrada una de ellas como alimentación emocional, y se definió como la ingesta alimentaria influenciada por emociones desagradables.

A partir del año 2010 la investigación sobre la AE tuvo un realce, en el que los estudios realizados han permitido ampliar la concepción, más allá de la visión psicodinámica, hacia una mirada encaminada a perspectivas de afrontamiento al estrés, en las cuales, este patrón alimentario, se concibe dentro de las estrategias focalizadas en la emoción, como: las de evitación y distracción, ello ante estados afectivos perturbadores [7,8]. Aunque también otras investigaciones apuntan que, el empleo de este patrón de alimentación se presenta como una forma de mejorar las experiencias emocionales positivas [2].

Ahora bien, entre los hallazgos encontrados sobre la elección de alimentos característicos en la $A E$ se ha identificado principalmente aquellos de tipo calóricos, dulces y grasos que, a razón de su efecto hedónico en el sistema de recompensa, refuerzan su consumo [9, 10,11]. En este sentido, a las personas que tienen una mayor inclinación por la utilización de este tipo de ingesta se les ha denominado como "comedores emocionales", con la particularidad de tener una mayor susceptibilidad al distrés, así como mayor sensibilidad neurofisiológica al refuerzo $[10,11]$.

Por otra parte, respecto a los efectos de la AE en la salud física y psicológica (principalmente en los comedores emocionales negativos), las primeras investigaciones apuntaban en exclusividad hacia un impacto en el peso corporal, como es en la obesidad, posteriormente a razón de su relación con la conducta alimentaria desinhibida, se comenzó a estudiar la relación de la $A E$ con los nombrados actualmente trastornos alimentarios y de la ingesta de alimentos (TAIA), identificando principalmente que este patrón alimentario puede fungir como un factor precipitante del trastorno por atracón (TPA); por otra parte, en pacientes que han sido sometidos a cirugía bariátrica se ha reportado que la $A E$ implica mayor interferencia en la pérdida de peso; cabe señalar que, este tipo de ingesta también se ha encontrado asociada con el síndrome metabólico [4,1218].

El impacto de la $A E$ en la salud física y psicológica en población adulta, cobra relevancia al revisar las estadísticas mundiales, principalmente en cuanto al sobrepeso y la obesidad, así como en los TAIA. En cuanto al exceso de peso, actualmente se ha estimado a nivel mundial un $39 \%$ y $13 \%$ de la población presentan sobrepeso y obesidad respectivamente [19], mientras que en México, la Encuesta Nacional de Salud y Nutrición de Medio Camino del 2016 [20] reportó que el 


\section{Hidalgo, Vol. 8, No. 15 (2019) 255-263}

$72.6 \%$ de adultos padecen alguna de estas condiciones 19,20. Respecto a los TAIA, la AE se ha relacionado principalmente con el TPA, el cual se considera la patología alimentaria más prevalente, estimándose en el Manual Diagnóstico y Estadístico de los Trastornos Mentales, en su quinta versión [21], que en los Estados Unidos la prevalencia en mujeres adultas es de $1.6 \%$ y en varones del $0.8 \%$. En México por su parte, en el año 2001 se reportó la presencia de bulimia nerviosa y trastornos de la conducta alimentaria no especificados (en los que se incluía el trastorno por atracón) fue del $0.24 \%$ y $0.91 \%$ respectivamente [22].

Si bien la investigación sobre alimentación emocional ha incrementado, poco se ha profundizado en la delimitación conceptual del constructo, por lo que el presente documento tiene como finalidad hacer una revisión del constructo a través de sus fundamentos teóricos, incluyendo las distintas acepciones con las que se cuenta y la forma en la que se evaluado psicométricamente, a partir de lo cual se plantean cuatro puntos de discusión para futuros estudios y planteamientos: (1) sobre la especificidad de la cantidad de alimento (aludiendo o no necesariamente a la sobreingesta) y grado de control, (2) en tanto a la elección y selección del alimento que se consume en estos episodios, (3) la consideración de emociones de distinta naturaleza y sobre (4) la traducción más idónea del nombre de este constructo al idioma en español que permitan una mejor comprensión de alimentación emocional y sus implicaciones. Los cuatro puntos mencionados previamente, se vuelven imprescindibles para investigaciones más sólidas y mediciones que reflejen con claridad el mismo constructo, puesto que actualmente se observa cierta ambigüedad en cada una de estas consideraciones, principalmente en las escalas psicométricas utilizadas para evaluar el constructo. De hecho, algunos estudios han evidenciado que los principales instrumentos utilizados para medir la $\mathrm{AE}$ carecen de validez predictiva, al ser contrastados con observaciones realizadas en ambientes controlados 0 naturalistas, sugiriendo que las puntuaciones obtenidas en los mismos, más que aludir a un incremento de la conducta alimentaria ante estados emocionales negativos, podrían estar reflejando variables como: preocupación por la comida, alimentación descontrolada, una tendencia a atribuir la sobreingesta a la experimentación de emociones negativas y alimentación reactiva [23].

\section{Alimentación emocional}

El constructo de alimentación emocional emergió de concepciones psicodinámicas que tenían como objetivo explicar la etiología de la obesidad bajo la consideración de factores psicológicos, siendo la teoría psicosomática la principal retomada para la denominación de este tipo de ingesta [4].

Poco tiempo después, Stunkard identificó tres patrones de la conducta alimentaria en personas con obesidad: el síndrome del comedor nocturno, el atracón y el denominado por el propio autor como "comer sin saciedad" [24]. En el caso de los dos primeros, se señala al estrés como un factor precipitante de la hiperfagia. Los estudios realizados por Stunkard brindaron las bases para la consolidación del término de trastorno por atracón, caracterizado por una ingesta excesiva en un corto periodo de tiempo seguido de sentimientos de culpa; en este sentido, desde el punto de vista del autor la sobreingesta es vista como una forma inconsciente de resolver un conflicto o malestar [24].

Por su parte, Slochower [25] hipotetizó que la AE de las personas con obesidad surgía cuando éstas desconocían la causa de su excitación emocional o no podían nombrarla con una etiqueta, a diferencia de las personas con normopeso, en quienes en situaciones símiles no se halló un incremento en su ingesta de alimentos [26]. Si bien, diferentes estudios de investigación continuaron desarrollándose en ambientes controlados donde se comparaban la reactividad emocional y la sobreingesta ante la inducción de distrés (comprendida esta ingesta excesiva como la $A E$ ) entre personas con obesidad y normopeso, fueron Lowe y Fisher [27] quienes realzaron la necesidad de prestar mayor atención a la alimentación emocional en personas con obesidad; los autores plantearon que la $A E$ como un factor explicativo de la sobreingesta 0 atracón y en sus resultados identificaron que puntajes más elevados indicativos de $\mathrm{AE}$ en mujeres estudiantes mujeres universitarias con obesidad en comparación con las que no presentaban exceso de peso; además se observó asociaciones de la $\mathrm{AE}$ con el consumo de alimentos clasificados como "refrigerios o bocadillos".

La conceptualización de alimentación emocional ha sido definida de manera más específica a través de los instrumentos psicométricos utilizados y creados para evaluarla. El primer antecedente se ha ubicado con el Cuestionario de Tres Factores de la Alimentación (TFEQ, por sus siglas en inglés) creado por Stunkard y Messick [6], el cual, evalúa tres estilos alimentarios poco saludables: restricción cognitiva en la alimentación, desinhibición del control de la alimentación y susceptibilidad al hambre. Aunque, como se puede apreciar, la $A E$ no estaba considerada en la versión original del instrumento, en una revisión realizada posteriormente por Ganley [3], se identificó que el factor de desinhibición, contenía dos agrupaciones de ítems o factores, a los cueles denominó: labilidad de peso y alimentación emocional. De esta manera, el cuestionario 
quedó conformado por cuatro factores: restricción cognitiva, susceptibilidad al hambre, labilidad del peso y alimentación emocional, destacando la importancia de este último factor, en la alimentación de personas con sobrepeso; algunos de los ítems que se incluyeron en esta subescala fueron: "cuando me siento ansioso, me descubro comiendo", "cuando me siento solo me consuelo comiendo" y "continúas atracándote aun cuando no tienes hambre"; este último reactivo como puede observarse, refiere particularmente a episodios de atracón. Un segundo análisis del TFEQ, llevado a cabo por Karlsson et al. [27] obtuvo una solución con las dimensiones: restricción cognitiva, alimentación descontrolada y alimentación emocional; esta última subescala se conformó por tres reactivos, dos de ellos son los retomados anteriormente en la revisión de Ganley además del ítem "cuando me siento triste, como a menudo en exceso", estos enunciados reflejan la conceptualización de la $\mathrm{AE}$ como el empleo de la sobreingesta de comida con la finalidad de disminuir el malestar experimentado por afectos desagradables (tales como la ansiedad, tristeza y soledad). Cabe señalar que el TFEQ está construido bajo el modelo de regulación de alimentos, el cual explica el rol de la restricción alimentaria como un factor desencadenante del atracón alimentario. En México, el TFEQ, fue evaluado en sus propiedades psicométricas en mujeres de entre 15 y 35 años de edad, análisis en el que se obtuvo únicamente dos de sus dimensiones originales: restricción alimentaria y desinhibición, sin figurar el factor de alimentación emocional [29].

El Cuestionario Holandés del Comportamiento Alimentario (DEBQ, por sus siglas en inglés) creado por van Strien et al. [5] evalúa la conducta alimentaria a partir de tres dimensiones alimentación restringida, emocional y externa, a partir de la consideración de las teorías de la alimentación restringida, la psicosomática y la de externalidad. De acuerdo con los autores del DEBQ, este instrumento surge en respuesta a la necesidad de comprender los patrones alimentarios de personas con obesidad, que pueden tener un rol importante en la aparición del sobrepeso. Si bien, en los tres patrones alimentarios se habla de sobreingesta, van Strien et al. distinguen la $A E$ de la alimentación externa, en función del estímulo precedente al consumo excesivo de alimentos. En el caso de la alimentación emocional esta conducta es señalada como el resultado de la experimentación de emociones (generalmente desagradables como: el enojo, o difusas como: la soledad, y el aburrimiento); mientras que en la alimentación externa la sobreingesta se propicia únicamente por las características de los alimentos (como: el olor y su aspecto). Pese a que la $A E$, dentro del $D E B Q$, es definida en términos de sobreingesta, cabe resaltar que los reactivos indagan exclusivamente sobre la intensidad del deseo de comida frente a estados anímicos negativos (con una escala de respuesta tipo Likert de cinco puntos, que va de uno nunca a cinco muy a menudo), sin involucrar necesariamente la ingesta de una gran cantidad de alimentos; algunos de sus reactivos son: “¿tienes deseo de comer cuando estás irritado?", "¿tienes deseo de comer cuando no tienes nada que hacer? y “¿tienes deseo de comer cuando estás ansioso, preocupado o tenso?" [5].

Posteriormente, Arnow, Kenardy y Agras [30] crearon la Escala de Alimentación Emocional (EES, por sus siglas en inglés), con la finalidad de facilitar la investigación sobre la relación existente entre la sobreingesta y los estados afectivos negativos. Los autores señalaron hallazgos sobre niveles altos de episodios de atracón vinculados con la experimentación del deseo de comer frente a emociones desagradables. Por otra parte, los autores del EES refirieron la necesidad de mejorar la evaluación de la $A E$, al señalar que el TFEQ carecía de una escala de respuesta tipo Likert y el DEBQ no permitía distinguir entre estados afectivos negativos específicos, ni la sobreingesta. A razón de ello, el EES quedó configurado con tres subescalas: enojo, ansiedad y depresión; en las cuales los ítems son únicamente términos alusivos a sentimientos pertenecientes a las dimensiones mencionadas, entre algunas de las palabras estímulo se encuentran: "resentido", "emocionado" y "celoso". En este instrumento se considera el grado de urgencia de comer (desde "sin deseo de comer" a "una urgencia abrumadora por comer").

Otro instrumento que se ha visto como una opción para evaluar la $\mathrm{AE}$ es el Cuestionario sobre Conducta Alimentaria Relacionada a Emociones y Estrés (EADES, por sus siglas en inglés) creado por Ozier et al. [31] con la finalidad de medir el uso de la comida para afrontar el estrés y las emociones. EI EADES toma como sustento teórico el Modelo Transaccional del Estrés y Afrontamiento [31]. Uno de los factores identificados en esta unidad de medida fue la alimentación relacionada con la emoción y el estrés; en este factor, de acuerdo con lo señalado por los autores, se identifica a la alimentación como una estrategia de afrontamiento posterior a la experimentación de ciertas emociones o de estrés, aludiendo a la $\mathrm{AE}$ como un aspecto de la sobreingesta; esta subescala considera reactivos vinculados a la autoeficacia, por ejemplo: "como cuando estoy ansioso", "estoy seguro que puedo controlar mi alimentación cuando estoy ansioso" y "no tengo control sobre la cantidad que como". El segundo factor del EADES es la evaluación de la habilidad y los recursos de afrontamiento, incluye ítems como: "soy capaz de conocer mis necesidades emocionales", "soy capaz de lidiar situaciones estresantes" y "tengo el control de mis 
emociones". El último factor fue nombrado como evaluación de estresores e influencias externas, en él se encuentran reactivos como: "me preocupo por lo que otras personas piensen de mi", "siento la necesidad de hacer felices a los demás" y "no veo los desafíos como estresantes". De acuerdo con los autores del EADES, estos factores pueden ayudar a comprender la conducta de sobreingesta o atracón. En la versión validada en población mexicana, se extrajeron las subescalas denominadas: (1) autoeficacia en la alimentación relacionada con la emoción y el estrés, incluyendo ítems como: "cuando estoy molesto conmigo mismo como", "no me puedo controlar cuando como" y "me consuelo con la comida", (2) autoconfianza en la alimentación relacionada con la emoción y el estrés, en la que algunos de los ítems incluidos son: "considero que puedo controlar mi alimentación cuando estoy frustrado", "considero que puedo controlar mi alimentación cuando estoy feliz" y "considero que puedo controlar mi alimentación cuando estoy feliz". Finalmente, el factor (3) evaluación de la habilidad y los recursos de afrontamiento, incluye reactivos como: "trato de resolver el problema cuando sé que hay algo mal en mi vida", "soy capaz de manejar situaciones estresantes" y "mi familia me apoya cuando tengo problemas" [32]. Las autoras de la validación del EADES señalan que, este instrumento puede ser útil para medir la conducta alimentaria emocional, aunque en otros estudios realizados por la autora principal se ha medido únicamente el primer factor para la evaluación de la $A E$ $[32,33]$. Es importante señalar que, en el estudio de propiedades psicométricas realizado en población mexicana, las dos primeras dimensiones identificadas fueron extraídas del primer factor de la escala original del EADES, que incluía reactivos alusivos a la evaluación del uso de alimentos frente a emociones tanto positivas como negativas (entre ellas: la ansiedad, el enojo, la tristeza, la frustración, el sentirse aliviado, etcétera), así como la habilidad de controlar la ingesta ante tales estados afectivos.

En México, la Escala de Factores de Riesgo Asociados con Trastornos de la Alimentación (EFRATA, versión adolescentes, jóvenes y adultos [34]) fue el primer instrumento creado y validado en población nacional que permitía evaluar la $\mathrm{AE}$, a partir del factor denominado "comer por compensación psicológica". De manera general, el EFRATA permite evaluar actitudes como conductas alimentarias anómalas, específicamente la subescala que mide la $A E$ fue definida por los autores como: una forma compulsiva de comer bajo la influencia del estado anímico. La evaluación del factor comer por compensación psicológica se conforma de cuatro ítems, entre ellos se encuentra: "siento que la comida me tranquiliza", "creo que la comida es un buen remedio para la tristeza o depresión" y "me sorprendo pensando en comida". Mientras que, en la versión adaptada para preadolescentes (EFRATA-II [35]), el factor alusivo a la $A E$ cambió su nombre original a "sobreingesta alimentaria por compensación psicológica”. Es relevante resaltar la modificación al nombre empleado para medir la $A E$ en la primera versión de la segunda, puesto que, en el EFRATA-II el factor de sobreingesta alimentaria por compensación psicológica agrupó una mayor cantidad de reactivos pertenecientes a dos factores de la conducta alimentaria (el de sobreingesta y el compulsivo) de la escala original. Cabe señalar que en la validación del EFRATA-II el factor de sobreingesta por compensación psicológica se conceptualizó como: la relación entre el estado de ánimo con una ingesta poco saludable, asociada a problemas de sobrepeso y obesidad. Esta última validación cuenta con versiones para cada sexo, en la de mujeres se encuentran ítems como: "soy de las que se atiborran de comida", "cuando estoy triste me da por comer" y "como sin control". Por su parte, en la versión para hombres, algunos de los ítems son: "siento que tengo más hambre cuando me enojo", "siento que podría comer sin parar" y "me sorprendo pensando en comida" [35].

La escala de $A E$ que se ha identificado como la más reciente para población mexicana es la de Alimentación Emocional construida por Rojas y García-Méndez [2]. En esta unidad de medida, se considera a la alimentación emocional en términos de "comer por apetencia, pero sin hambre fisiológica, en respuesta a acontecimientos tanto positivos como negativos, con la finalidad de evitar, regular, enfrentar o mejorar tal experiencia emocional" ( $p$. 90). Este instrumento, mide la $A E$ a través de cinco dimensiones: (1) emoción, la cual refiere a modificaciones en la alimentación con la finalidad de regular; ya sea la intensidad o duración de la vivencia de ciertos estados afectivos, algunos de sus reactivos son: "cuando tengo problemas ingiero más de lo normal", "después de pasar un susto me da hambre" y "cuando me emociono me da hambre"; (2) familia, es decir el comer por demostración de afecto y simbolismo emocional en el núcleo familiar, entre los reactivos se encuentran "comer es un placer que se disfruta más en familia", "la hora de la comida es sagrada" y "cuando me preparan de comer siento que me quieren"; (3) indiferencia, alude al escaso interés por aspectos nutricionales de los alimentos y cuidado en la ingesta, algunos ítems son: "pongo poca atención en el contenido nutricional de lo que como", "me preocupo poco por mi alimentación" y "cuando tengo hambre ingiero cualquier alimento"; (4) cultura, refleja el grado en el que conductas alimentarias se aceptan y comparten en los miembros del grupo de pertenencia, ejemplos de reactivos en este factor son: "es común que estando en fiestas coma más 
de lo normal", "como más de lo normal cuando asisto a eventos familiares" y "como más de lo normal cuando asisto a reuniones con los amigos"; finalmente la dimensión (5) efecto del alimento, señala las modificaciones en el ánimo previamente, durante y post ingesta de alimentos, algunos de los ítems son: "cuando tengo hambre, mi estado de ánimo cambia", "después de comer, mi estado de ánimo cambia" y "comer mejora mi estado de ánimo" [2]. Llama la atención que, en el cuestionario de alimentación emocional de Rojas y García-Méndez, no se aprecia tan visiblemente el aspecto del "control", el cual sí es considerado en otros instrumentos como el EADES y EFRATA-II.

Ahora bien, los precedentes teóricos de la $A E$ que han sido sustento, tanto para la construcción de instrumentos psicométricos como para diversas investigaciones científicas son retomados en el siguiente apartado.

\section{Antecedentes teóricos de la alimentación emocional}

El estudio de la alimentación emocional tiene sus raíces en teorías e investigaciones encaminadas a explicar la obesidad, a través de la desinhibición y sobreingesta alimentaria, desde una visión psicodinámica.

\section{Teoría psicosomática}

El concepto de $A E$, emergió del planteamiento de la relación estrecha de las emociones con la conducta alimentaria. La principal teoría que se ha retomado en el estudio de la alimentación emocional es la psicosomática, la cual explica que el desarrollo y mantenimiento de la obesidad, a partir de factores psicológicos, específicamente se alude a la hiperfagia como resultado del displacer experimentado ante estados afectivos desagradables como: el miedo, la ansiedad, la tristeza y/o el enojo. La sobreingesta alimentaria de personas con obesidad es vista como una estrategia para decrementar el malestar suscitado por estados afectivos desagradables, consiguientemente, dicho empleo de la comida como defensa, favorece la hiperfagia y por ende el exceso de peso [4]. Un autor que ha aportado a esta teoría es Bruch, quien menciona que las personas que presentan obesidad son una población mayormente vulnerable a ingerir alimentos frente al distrés, debido a experiencias tempranas en las que la cuidadora primaria alimentó al infante sin discriminar el motivo del llanto (por hambre u otras necesidades), lo que pudo generar, en estos menores de edad, un fallo en el aprendizaje discriminativo de sensaciones internas (estado fisiológico hambre-saciedad) con otras señales de malestar emocional; esta situación trasciende en las diferentes desarrollo y puede resultar en una mayor ingesta de alimentos en respuesta a estados emocionales desagradables [36].

\section{Teoría interna-externa de la obesidad}

Desde la teoría interna-externa de la obesidad se plantea que las personas con obesidad exhiben una respuesta mayor a señales de comida que se encuentran en el ambiente, en comparación con las personas de un peso esperable para sus características; además las personas con exceso de peso presentan una percepción disminuida de las señales internas de hambre. Ambas condiciones, sitúan a las personas con obesidad en una posición de mayor susceptibilidad a la sobreingesta alimentaria. Cabe señalar, que desde esta teoría no es muy relevante el rol dado a los estímulos emocionales, únicamente se les ve como algunos de los factores precipitantes de la desinhibición en la conducta alimentaria [37].

\section{Teoría del set point de Nisbett}

En la teoría del set point, se explica que cada cuerpo tiene una predisposición a mantener determinado peso, el cual en las personas con obesidad suele estar situado biológicamente en cifras superiores a lo "normal" o esperable. Dentro de un contexto sociocultural, en el que el concepto de la belleza tiende a la cultura de la delgadez (principalmente en el caso de las mujeres), la condición del exceso de peso puede incidir en el desarrollo de ciertas conductas alimentarias no saludables de personas con sobrepeso u obesidad, a favor de un decremento en su ingesta alimentaria necesaria. Tras la práctica de dietas restrictivas en estas personas, el autor de esta teoría sugiere que se pone en marcha una respuesta metabólica para volver al peso corporal establecido genéticamente, haciendo al individuo mayormente susceptible a las señales de alimento encontradas en el ambiente, y también manifiestan una emotividad incrementada, que conduciría a la sobreingesta. Todo este proceso de respuesta tendría como objetivo de volver al llamado punto de regulación de peso (set point) [38].

\section{Teoría de la alimentación restringida}

En sintonía con la teoría del set point [38], la teoría de la restricción alimentaria argumenta que la conducta de ingesta emocional y/o por estímulos externos, se deriva de dietas restrictivas intensas, originadas en buena medida por presiones sociales. Estas dietas tienen como resultado un hambre persistente que, en un momento de pérdida de autocontrol en situaciones como la experimentación de afectos desagradables o estímulos externos, la conducta alimentaria se ve desinhibida [3943]. 


\section{Teoría del escape}

La teoría del escape, explica el uso de los alimentos como una forma de reducir el malestar emocional, particularmente en lo que a conducta de atracón respecta. Esta perspectiva indica que, en personas que se manejan con altos niveles de estándares, expectativas y autoexigencias, se presenta una mayor sensibilidad a las demandas provenientes de los demás y del exterior en general, y que ante situaciones en que no se consigue cumplir con dichas demandas, se presenta una elevada autocrítica, que genera angustia emocional. La experimentación de dicha angustia es afrontada con un aumento en la ingesta de alimentos, descrita por los autores, como una forma de "escape" que logra cambiar el foco de atención a otro estímulo no aversivo [44].

\section{Teorías conductuales}

Las teorías conductuales explican el comportamiento, como un fenómeno observable, a partir de la asociación de los elementos: estímulo y respuesta [45]. De manera específica, Bongers y Jansen [46] han retomado el condicionamiento clásico para brindar una posible explicación de la alimentación emocional. Estas autoras comprobaron, en muestras de estudiantes universitarios (de entre 18 a 30 años), que se podía condicionar el apetito frente estados emocionales negativos, fungiendo estos últimos como estímulos condicionados que suscitaban como respuestas: las expectativas y un mayor deseo de alimento. Otro resultado obtenido en el mismo estudio fue que, aquellos participantes con un índice de masa corporal (IMC) más alto tenían una preferencia mayor por la recompensa alimentaria (chocolate) en comparación con la preferencia monetaria, en contraste los participantes con un IMC más bajo manifestaron una mayor preferencia monetaria. A partir de estos hallazgos, las autoras sugieren que las personas con exceso de peso pueden tener una mayor susceptibilidad emocional a las señales de comida.

\section{Conclusiones}

La alimentación emocional es un constructo que emergió desde el enfoque psicodinámico, que dé inicio buscaba explicar la obesidad bajo la consideración de factores psicológicos. Una de las teorías desde esta perspectiva es la psicosomática (principal antecedente referido en estudios de la $A E$ ), en la cual se señala el poder de la angustia emocional, como un agente modificador, en la conducta alimentaria de personas con exceso de peso, en quienes el empleo de comida es visto como un mecanismo de afrontamiento ante el malestar emocional experimentado, y que a su vez favorece y mantiene el exceso de peso [4].

La presente revisión de teorías, que han sido señaladas como precedentes de la $\mathrm{AE}$, exhibe coincidencias en la mayoría de ellas, principalmente en los siguientes aspectos: (1) señalar a los estados afectivos desagradables como fuentes potenciales para modificar la conducta alimentaria, (2) el empleo de alimentos como un mecanismo de afrontamiento ante el estado afectivo experimentado, (3) que las personas presenten una capacidad interoceptiva disminuida y, (4) una mayor susceptibilidad a presentar episodios de sobreingesta debido a la autoimposición de dietas restrictivas [4,3643].

Posteriormente, diversos autores que se han dado a la tarea de diseñar instrumentos psicométricos sobre $\mathrm{AE}$, en el proceso de definir conceptualmente el constructo, también han contribuido a diferenciar este tipo de ingesta de otros patrones alimenticios; por ejemplo, al distinguir la alimentación externa de la emocional, si bien ambas convergen en referir a una sobreingesta, estos patrones alimentarios se distinguen a razón del estímulo que la induce, en el caso de la $A E$ la ingesta excesiva se debe a estados emocionales perturbadores, que son "combatidos" por el empleo de comida (en algunas teorías, como las conductuales señalan que la AE puede deberse a un aprendizaje por asociación), mientras que en la alimentación externa la sobreingesta se debe exclusivamente a características del alimento. Es entonces que, partiendo de conocer los antecedentes teóricos de la $A E$, se comprende su conceptualización bajo la consideración de sobreingesta o atracón en algunos instrumentos psicométricos, sin embargo, la observación de los reactivos que las constituyen, no siempre reflejan la desinhibición alimentaria, sino que aluden en la mayoría de veces al empleo de la comida como una estrategia para lidiar con ciertas emociones. Esta discrepancia entre lo conceptual y operacional, permite poner a discusión (1) si necesariamente debe presentarse la sobreingesta y/o pérdida de control para que se le considere $A E$ o bien, el simple uso de los alimentos como forma de lidiar con el estrés y otras emociones es suficiente para considerar que se presenta este tipo de ingesta. Actualmente algunas investigaciones han reportado que la $A E$ no es exclusiva de IMC elevados, lo que podría sugerir que la $A E$ no siempre está ligada a episodios de atracón [3,5,7]. Por otro lado, Bongers y Jansen [47] han sugerido que la alimentación emocional podría ser indicativo de una sobreingesta en general de ciertas personas, aludiendo al término "comedores reactivos" como un sustituto del término de comedores emocionales.

$\mathrm{Si}$ bien las investigaciones han encontrado que los comedores emocionales se caracterizan por elecciones 
de alimentos poco saludables, a nivel conceptual y operacional no se hace alusión a ello, por lo que valdría la pena preguntarse (2) qué tanto la elección de los tipos de alimentos (por ejemplo: dulces, salados, amargos, picantes, grasos o con alto contenido en fibra) es independiente, mientras se empleen como una estrategia de afrontamiento, incluso si se le continuaría considerando una estrategia disfuncional o no a partir del contenido nutricional del alimento [48-49].

Otro aspecto relevante a señalar es sobre la naturaleza de las emociones en la $\mathrm{AE}$, puesto que, si bien en las teorías revisadas se habla en exclusividad de afectos desagradables, en algunas escalas se toma en cuenta a las emociones positivas. En el EADES [32] dicha consideración se refleja en dos de sus ítems: "considero que puedo controlar mi alimentación cuando me libero de preocupaciones" y "considero que puedo controlar mi alimentación cuando me siento feliz", así como en la Escala de Alimentación Emocional, con reactivos como: "comer es un placer que se disfruta más en familia" y "comer en familia es muy importante para mí". Al respecto, es importante señalar que en ambos instrumentos no queda muy claro si necesariamente se trata de $\mathrm{AE}$, puesto que en el primer caso se centra en la variable control que en las definiciones no se señala como parte de la AE y por otra parte, en el caso del último instrumento, sus reactivos evalúan el uso de la comida como parte de la cultura en la que la persona se encuentra inmiscuido. A razón de ello cabe reflexionar y cuestionar (3) en qué medida la definición conceptual debe incluir todas las emociones, independientemente de su naturaleza tanto positivas como negativas [31,35].

Finalmente, un aspecto relevante a poner sobre la mesa, para futuros análisis, es referente a la traducción en español del término original emotional eating, del cual se han identificado tres: alimentación emocional, comer por compensación psicológica y sobreingesta alimentaria por compensación psicológica; términos que si bien aluden al empleo de alimentos para reducir o modificar un estado afectivo desagradable, los últimos dos se diferencian en que el nombre de sobreingesta alimentaria por compensación psicológica, involucra la consideración de un consumo de alimentos caracterizado por ser compulsivo y excesivo, el cual podría implicar la presencia de $A E$ únicamente en casos en lo que se presenten episodios de atracón, a raíz de ello cabe cuestionar sobre (4) cuál de los términos en español antes mencionados, se apega más al constructo original de "emotional eating" [2, 34, 35].

Precisamente, a partir de las necesidades delimitativas que parece requerir el constructo de $\mathrm{AE}$, algunos autores han señalado inconsistencias en la capacidad predictiva y validez discriminativa en sistemas de medición de la $A E$, mencionando que los resultados de estas escalas podrían estar indicando preocupaciones alimentarias en general, falta de control, atribución de las emociones a la sobreingesta, sugiriendo así la conveniencia de contar con medidas más objetivas [7,23].

A razón de lo anteriormente señalado, se refleja la complejidad y necesidad de tener una delimitación más clara del constructo de alimentación emocional, así como su evaluación y creación de modelos explicativos, que permitan una mayor comprensión de la $A E$, así como una distinción más clara frente a constructos como la desinhibición/atracón. Por lo que la investigación, en este sentido, tiene un amplio campo de estudio para favorecer la implementación de intervenciones que contribuyan a decrementar la prevalencia de patologías como el sobrepeso y la obesidad, el síndrome metabólico, los TAIA, etcétera [12-18].

\section{Agradecimientos}

El presente artículo fue realizado gracias al apoyo recibido por parte del Consejo Nacional de Ciencia y Tecnología (CONACYT) con la beca número 920841 para la realización de estudios de posgrado en el programa de Maestría en Ciencias Biomédicas y de la Salud de la Universidad Autónoma del Estado de Hidalgo.

\section{Referencias}

[1] Álvarez M, Odero D. Análisis dimensional de la conducta alimentaria desde el paradigma psicosomático. Interpsiquis. 2008;1:1-9.

[2] Rojas AT, García-Méndez M. Construcción de una Escala de Alimentación Emocional. Rev Iberoam Diagnóstico y Evaluación. 2017;3(45):85-95.

[3] Ganley RM. Emotional eating and how it relates to dietary restraint, disinhibition, and perceived hunger. Int J Eat Disord. 1988;7(5):63547.

[4] Kaplan HI, Kaplan HS. The psychosomatic concept of obesity. J Nerv Ment Dis. 1957;125(2):181-201.

[5] van Strien T, Frijters JER, Bergers GPA, Defares PB. The Dutch Eating Behavior Questionnaire (DEBQ) for assessment of restrained, emotional, and external eating behavior. Int $\mathrm{J}$ Eat Disord. $1986 ; 5(2): 295-315$.

[6] Stunkard AJ, Messick S. The three-factor eating questionnaire to measure dietary restraint, disinhibition and hunger. J Psychosom Res. 1985;29(1):71-83.

[7] Bongers P, Jansen A. Emotional Eating Is Not What You Think It Is and Emotional Eating Scales Do Not Measure What You Think They Measure. Front Psychol. 2016;7(1932).

[8] Spoor STP, Bekker MHJ, Van Strien T, van Heck GL. Relations between negative affect, coping, and emotional eating. Appetite. 2007;48(3):368-76.

[9] Camilleri GM, Méjean C, Kesse-Guyot E, Andreeva VA, Bellisle F, Hercberg S, et al. The Associations between Emotional Eating and 
Consumption of Energy-Dense Snack Foods Are Modified by Sex and Depressive Symptomatology. J Nutr. 2014;144(8):1264-73.

[10] van Strien T, Herman CP, Anschutz DJ, Engels RCME, de Weerth C. Moderation of distress-induced eating by emotional eating scores. Appetite. 2012;58(1):277-84.

[11] Leigh Gibson E. Emotional influences on food choice: Sensory, physiological and psychological pathways. Physiol Behav. 2006;89(1):53-61.

[12] Sanchez-Ruiz MJ, El-Jor C, Abi Kharma J, Bassil M, Zeeni N. Personality, emotion-related variables, and media pressure predict eating disorders via disordered eating in Lebanese university students. Eat Weight Disord. 2017;24(2):313-22.

[13] Wilkinson LL, Rowe AC, Robinson E, Hardman CA. Explaining the relationship between attachment anxiety, eating behaviour and BMI Appetite. 2018;127: 214-22.

[14] Bourdier L, Morvan Y, Kotbagi G, Kern L, Romo L, Berthoz S. Examination of emotion-induced changes in eating: A latent profile analysis of the Emotional Appetite Questionnaire. Appetite. 2018;123: $72-81$.

[15] Canetti L, Berry EM, Elizur Y. Psychosocial predictors of weight loss and psychological adjustment following bariatric surgery and a weightloss program: The mediating role of emotional eating. Int J Eat Disord. 2009;42(2):109-17.

[16] Rotella F, Fioravanti G, Godini L, Mannucci E, Faravelli C, Ricca V. Temperament and emotional eating: A crucial relationship in eating disorders. Psychiatry Res. 2015;225(3):452-7.

[17] Gómez-Peresmitré G, Acosta García V, Gorischnik R, Cuevas Renaud C, Pineda García G, Platas Acevedo RS, et al. Un estudio preliminar de los factores predisponentes de la conducta de atracón en tres culturas: México, Argentina y España. Rev Mex Trastor Aliment. 2013;4(2):68-78.

[18] Vera B, Dashti HS, Gómez-Abellán P, Hernández-Martínez AM, Esteban A, Scheer FAJL, et al. Modifiable lifestyle behaviors, but not a genetic risk score, associate with metabolic syndrome in evening chronotypes. Sci Rep. 2018;8(1):945.

[19] Organización Mundial de la Salud. Obesidad y sobrepeso. 2017.

[20] Secretaría de Salud. Encuesta Nacional de Salud y Nutrición de Medio Camino 2016. México; 2016

[21] American Psychiatric Association. Manual Diagnóstico y Estadístico de los Trastornos Mentales DSM-5. 5a ed. Arlington, VA: Panamericana; 2014.

[22] Mancilla-Díaz JM, Perezbolde VC, Franco K, Vázquez AR, López X, Álvarez G, et al. Prevalence of eating disorders in México.Featuring abstracts from the International Conference of Eating Disorders. Int J Eat Disord. 2004;35(4):489.

[23]. Bongers P, de Graaff A, Jansen A. "Emotional" does not even start to cover it: Generalization of overeating in emotional eaters. Appetite. 2016;7(1932).

[24] Stunkard AJ. Esating patterns and obesity. Psychiatr Q. 1959; $33: 284-92$

[25] Slochower J. Emotional labeling and overeating in obese and normal weight individuals. Psychosom. Med. 1976; 38: 131-139.

[26]. Slochower J, Kaplan SP. Anxiety, perceived control, and eating in obese and normal weight persons. Appetite. 1980; 1:75-83

[27] Lowe MR, Fisher EB. Emotional reactivity, emotional eating, and obesity: A naturalistic study. J Behav Med. 1983;6(2):135-49.

[28] Karlsson J, Persson L-O, Sjöström L, Sullivan M. Psychometric properties and factor structure of the Three-Factor Eating Questionnaire (TFEQ) in obese men and women. Results from the Swedish Obese Subjects (SOS) study. Int J Obes. 2000;24(12):171525 .
[29] López-Aguilar X, Mancilla-Díaz JM, Vázquez-Arévalo R, FrancoParedes K, Alvarez-Rayón GL, Téllez-Girón MT. Propiedades psicométricas del Cuestionario de Tres Factores de la Alimentación (TFEQ). Rev Mex Trastor Aliment. 2011;2(1):24-32.

[30] Arnow B, Kenardy J, Agras WS. The Emotional Eating Scale: the development of a measure to assess coping with negative affect by eating. Int J Eat Disord. 1995;18(1):79-90.

[31]. Ozier AD, Kendrick OW, Knol LL, Leeper JD, Perko M, Burnham J. The Eating and Appraisal Due to Emotions and Stress (EADES) Questionnaire: Development and Validation. J Am Diet Assoc. 2007;107(4):619-28.

[32] Lazarevich I, Irigoyen-Camacho ME, del Consuelo Velázquez-Alva M, Salinas-Ávila J. Psychometric characteristics of the Eating and Appraisal Due to Emotions and Stress Questionnaire and obesity students. Nutr Hosp. 2015;31(6):2437-44.

[33]. Lazarevich I, Irigoyen Camacho ME, Velázquez-Alva M del C, Zepeda Zepeda M. Relationship among obesity, depression, and emotional eating in young adults. Appetite [Internet]. 2016 Dec 1 [cited 2018 Aug 16];107: 639-44.

[34] Gómez-Peresmitré, G. (2001). Factores de riesgo en trastornos de la conducta alimentaria. Teoría práctica y prevalencia en muestras mexicanas. Conferencia Magistral de los 450 años de la Universidad, UNAM.

[35] Platas RS, Gómez-Peresmitré G. Propiedades psicométricas de la Escala de Factores de Riesgo Asociados con Trastornos de la Alimentación (EFRATA-II) en preadolescentes mexicanos. Psicol y Salud. 2013;23(2):251-9.

[36] Bruch H. Eating Disorders: Obesity, Anorexia Nervosa and the Person Within. New York: Basic Books; 1973.

[37] Schachter S. Obesity and eating. Science. 1968;161(3843):751-6.

[38] Nisbett RE. Hunger, obesity, and the ventromedial hypothalamus. Psychol Rev. 1972;79(6):433-53.

[39] Herman CP, Polivy J. Restrained eating. En: Stunkard, A. J. (Ed.). Obesity. Philadelphia, London,Toronto:Saunders; 1980.208-225.

[40] Polivy J, Herman CP. Breaking the Diet Habit: The Natural Weight Alternative. New York: Basic Books;1983.

[41] Polivy J, Herman CP. The effects of alcohol on eating behavior: Disinhibition or sedation? Addictive Behaviors.1976; 1: 121-126.

[42] Herman CP, Polivy J. Anxiety, restraint and eating behavior. journal of Abriormal Psychology.1975; 84: 666-672.

[43] Polivy J, Herman CP. Restrained Eating and Food Cues: Recent Findings and Conclusions. Curr Obes Rep. 2017;6(1):79-85.

[44] Heatherton TF, Baumeister RF. Binge Eating as Escape From SelfAwareness. Psychological Bulletin. 1991;110

[45]Schunk D. Teorías del aprendizaje. Una perspectiva educativa. México: Pearson; 2012.

[46] Bongers P, Jansen A. Emotional eating and Pavlovian learning: evidence for conditioned appetitive responding to negative emotional states. Cognition and Emotion. 2015.

[47] Rojas AT. Alexitimia, funcionamiento familiar y estilo de vida: predictores de la alimentación emocional. Universidad Nacional Autónoma de México; 2016.

[48] Camilleri GM, Méjean C, Kesse-Guyot E, Andreeva VA, Bellisle F, Hercberg S, et al. The Associations between Emotional Eating and Consumption of Energy-Dense Snack Foods Are Modified by Sex and Depressive Symptomatology. J Nutr. 2014;144(8):1264-73.

[49] De Lauzon B, Romon M, Deschamps V, Lafay L, Borys J-M, Karlsson J, et al. The Three-Factor Eating Questionnaire-R18 Is Able to Distinguish among Different Eating Patterns in a General Population. J Nutr. 2004;134(9):2372-80. 